\title{
Vibrational Frequencies of Semirigid Molecules: A General Method and Values for Ethylbenzene ${ }^{1}$
}

\author{
By William J. Taylor ${ }^{2}$ and Kenneth S. Pitzer ${ }^{3}$
}

\begin{abstract}
It is shown that in normal coordinate calculations of the vibrations of molecules it is sometimes advantageous to calculate the kinetic-energy matrix, rather than the reciprocal kinetic-energy matrix. Explicit formulas are given for the elements of the kinetic-energy matrix. Illustrative calculations are given for propane, toluene, and ethylbenzene. A semiempirical assignment of the vibration frequencies of ethylbenezene is made on the basis of these calculations and the spectroscopic data.
\end{abstract}

\section{Introduction}

For the purpose of calculating the thermodynamic functions (particularly the entropy) of molecules by statistical methods, it is important that the low frequencies of vibration be assigned as accurately as possible. When these frequencies have not been observed in the spectra, the assignment must be made either by analogy with other molecules, or on the basis of a normal coordinate calculation, using force constants determined from other molecules. Even when the frequencies have been observed, a preliminary assignment by analogy or calculation is often necessary for the identification of the observed frequencies. However, a complete normal coordinate analysis for molecules with many atoms is so time-consuming as to be impracticable in most investigations. Therefore, an approximate method of calculating the low skeletal frequencies of large molecules should be of considerable value in connection with thermodynamic calculations.

\footnotetext{
1 This investigation was performed at the National Bureau of Standards as part of the work of the American Petroleum Institute Research Project 44 on the "Collection, Analysis, Calculation, and Compilation of Data on the Properties of Hydrocarbons".

${ }^{2}$ Research Associate on the American Petroleum Institute Research Project 44 at the National Bureau of Standards.

${ }^{3}$ Associate Supervisor, American Petroleum Institute Research Project 44; Professor of Chemistry, University of California, Berkeley, Calif.
}

In this paper there is described an approximate method that is based on the replacement of the actual molecule by a semirigid model (of the same dimensions and masses) in which certain groups of atoms move as rigid units. In this way the number of internal coordinates of the molecule may be reduced sufficiently to make the calculation of the low skeletal frequencies practicable. The semirigid model should be satisfactory, provided that all the frequencies of internal motion of the groups assumed to be rigid are either considerably higher than the frequencies to be calculated, or else do not interact seriously with them because of molecular symmetry or other factors. In order to reduce the error due to interactions, it is desirable that the necessary force constants be determined by analogous (semirigid model) calculations on other molecules in which the frequencies are known.

The analysis given in this paper shows that the kinetic-energy matrix of a semirigid molecule is usually more easily calculated than the reciprocal kinetic-energy matrix. The reverse is true for a complete normal coordinate calculation for the molecule. Formulas are given that reduce the calculation of the kinetic-energy matrix to a consideration of the masses, moments of mass, and moments of inertia of the rigid groups. 
An illustrative calculation is given for ethylbenzene by using force constants determined from propane and toluene. A semiempirical assignment of the vibrational frequencies of ethylbenzene is made on the basis of this calculation and the spectroscopic data. These calculations for ethylbenzene were completed in September 1945, were and used in the tables of the thermodynamic properties of ethylbenzene issued November 30, 1945, by the American Petroleum Institute Research Project 44.

\section{Mathematical Analysis}

\section{Kinetic Energy of Semirigid Molecules}

The first step in the normal coordinate analysis of a vibrating system is the computation of the kinetic-energy matrix, or of its reciprocal. Certain general features of the calculation will first be considered. Let the system of $n$ particles be characterized by $3 n$ generalized coordinates, $q$, six of which refer to over-all translation and rotation of the system (external coordinates), and the remainder of which specify the configuration of the system (internal coordinates). The present discussion is concerned only with small distortions of the system from its equilibrium configuration, so that the internal coordinates may pe assumed to measure this distortion and to have the value zero at the equilibrium configuration.

The kinetic energy is a quadratic form in the coordinate velocities, the $\dot{q}^{\prime}$ s,

$$
2 T=\tilde{\dot{q}} S \dot{q} .
$$

In this equation $\dot{q}$ is a column matrix, $\tilde{\dot{q}}$ is a row matrix (the transpose of $\dot{q}$ ), and $S$ is the square symmetric kinetic-energy matrix. In general, the elements of $S$ are functions of the $q$ 's (not of the $\dot{q}$ 's), but because of the restriction to configurations near the equilibrium configuration, $S$ is to be regarded as a constant matrix here.

For reasons that will appear presently, it will be convenient to assume that the coordinates are of three types, and to partition the column matrix $q$ accordingly.

$$
q=\left[\begin{array}{l}
q_{1} \\
q_{2} \\
q_{3}
\end{array}\right]
$$

The kinetic-energy matrix, $S$ may be partitioned into the corresponding submatrices, and it will also be convenient to designate by $G$ the reciprocal of $S$ and to partition $G$,

$S=\left[\begin{array}{lll}S_{11} & S_{12} & S_{13} \\ \tilde{S}_{12} & S_{22} & S_{23} \\ \tilde{S}_{13} & \tilde{S}_{23} & S_{33}\end{array}\right], G=S^{-1}=\left[\begin{array}{ccc}G_{11} & G_{12} & G_{13} \\ \widetilde{G}_{12} & G_{22} & G_{23} \\ \widetilde{G}_{13} & \widetilde{G}_{23} & G_{33}\end{array}\right]$

The concept of a semirigid molecule, with which this paper is principally concerned, will now be introduced. A semirigid molecule, as defined here, is one in which some of the internal coordinates, say the group $q_{3}$, remain zero throughout the molecular motion, so that $q_{3}=0$, and $\dot{q}_{3}=0$. Thus, if parts of the molecule move as rigid units in their equilibrium configurations, the internal coordinates of these parts will remain zero.

The kinetic-energy matrix for a semirigid molecule reduces to

$$
\left[\begin{array}{ll}
S_{11} & S_{12} \\
\tilde{S}_{12} & S_{22}
\end{array}\right]
$$

as $\dot{q}_{3}=0$. The equivalent expression for the kinetic energy is

$$
2 T=\tilde{\dot{q}}_{1} S_{11} \dot{q}_{1}+2 \tilde{\dot{q}}_{1} S_{12} \dot{q}_{2}+\tilde{\dot{q}}_{2} S_{22} \dot{q}_{2} .
$$

The form of the potential-energy expression will now be considered. As $q_{3}=0$, these coordinates do not influence the variable part of the potential energy (the assumption that $q_{3}=0$ is equivalent to setting the force constants for these coordinates equal to infinity). In addition, there will be a group of coordinates, say $q_{1}$ that do not occur in the expression for the potential energy, although $\dot{q}_{1}$ occurs in the kinetic energy. This group of coordinates of zero frequency includes the external coordinates and possibly (as an approximation) some very low frequency internal coordinates, in particular torsional motions. Finally, it will be assumed that the potential energy is a quadratic form in the remaining coordinates (harmonic vibrations) so that,

$$
2 V=\tilde{q}_{2} F_{22} q_{2},
$$

where $F_{22}$ is the symmetric potential-energy matrix.

If the Lagrangian equations of a system for which the kinetic and potential energies are given by eq 5 and 6 , respectively, are to be integrable, it is necessary to remove by a suitable 
transformation the cross term between $q_{1}$ and $q_{2}$ in the kinetic energy. The existence of this cross term is a consequence of the fact that the initial coordinates, $q_{1}$ have been defined in such a way that their conjugate momenta, $p_{1}=\left(\partial T / \partial \dot{q}_{1}\right)$, are not independent of $\dot{q}_{2}$. As a result, the momenta $p_{1}$ are not conserved, as they should be, because the coordinates $q_{1}$ do not enter into the potentialenergy expression, and are therefore not acted upon by any forces.

As it is desired to find the proper kinetic-energy matrix for the (arbitrary) internal coordinates $q_{2}$, it follows that the transformation should leave these coordinates unchanged. The most general linear transformation of this type is

$$
\left[\begin{array}{l}
q_{1}^{\prime} \\
q_{2}
\end{array}\right]=\left[\begin{array}{ll}
T_{11} & T_{12} \\
0 & E_{22}^{\prime}
\end{array}\right]\left[\begin{array}{l}
q_{1} \\
q_{2}
\end{array}\right],
$$

where $E_{22}$ is the unit matrix with the appropriate number of rows and columns. The matrices $T_{11}$ and $T_{12}$ are subject only to the condition that the transformed kinetic-energy matrix have the form

$$
\left[\begin{array}{ll}
S_{11}^{\prime} & 0 \\
0 & S_{22}^{\prime}
\end{array}\right]
$$

The equivalent expression for the kinetic energy is

$$
2 T=\tilde{\dot{q}}_{1}^{\prime} S_{11}^{\prime} \dot{q}_{1}^{\prime}+\tilde{\dot{q}}_{2} S_{22}^{\prime} \dot{q}_{2}
$$

The transformed kinetic-energy matrix is obtained by multiplying the original kinetic-energy matrix from the right by the reciprocal of the transformation matrix, and from the left by the transpose of the reciprocal (congruent transformation). Therefore,

$$
\begin{aligned}
& {\left[\begin{array}{ll}
S_{11}^{\prime} & 0 \\
0 & S_{22}^{\prime}
\end{array}\right]=\left[\begin{array}{cc}
\tilde{T}_{11}^{-1} & 0 \\
-\widetilde{T}_{12} \widetilde{T}_{11}^{-1} & E_{22}
\end{array}\right]} \\
& {\left[\begin{array}{ll}
S_{11} & S_{12} \\
\tilde{S}_{12} & S_{22}
\end{array}\right]\left[\begin{array}{cc}
T_{11}^{-1} & -T_{11}^{-1} T_{12} \\
0 & E_{22}
\end{array}\right]}
\end{aligned}
$$

Equation 10 is equivalent to the relations

$$
\begin{aligned}
S_{11}^{\prime}= & \tilde{T}_{11}^{-1} S_{11} T_{11}^{-1} \\
0= & \tilde{T}_{11}^{-1}\left(S_{12}-S_{11} T_{11}^{-1} T_{12}\right) \\
0= & \left(\tilde{S}_{12}-\tilde{T}_{12} \tilde{T}_{11}^{-1} S_{11}\right) T_{11}^{-1} \\
S_{22}^{\prime}= & S_{22}-\tilde{T}_{12} \tilde{T}_{11}^{-1} S_{12}-\tilde{S}_{12} T_{11}^{-1} T_{12}+ \\
& \tilde{T}_{12} \tilde{T}_{11}^{-1} S_{11} T_{11}^{-1} T_{12}
\end{aligned}
$$

Equations 12 and 13 are equivalent, and yield

$$
T_{11}^{-1} T_{12}=S_{11}^{-1} S_{12}
$$

as the condition that there be no cross term between $\dot{q}_{1}^{\prime}$ and $\dot{q}_{2}$ in the kinetic energy.

Equation 15 does not determine $T_{11}$ or $T_{12}$ separately, but only the product $T_{11}^{-1} T_{12}$, and therefore $S_{11}^{\prime}$ is not uniquely determined. However, $S_{22}^{\prime}$ is unique, as the combination of eq 14 and 15 yields

$$
S_{22}^{\prime}=S_{22}-\tilde{S}_{12} S_{11}^{-1} S_{12}
$$

The matrix $S_{22}^{\prime}$ will be called the reduced kineticenergy matrix for the internal coordinates (excluding those that do not occur in the expression for the potential energy). A result equivalent to eq 16 has been obtained by Majantz $[1]^{4}$ by an argument less fundamental than that presented here.

When applied to the external coordinates, the transformation of eq 7 is equivalent to the application of the conditions that the linear and angular momenta of the entire molecule are independent of the $\dot{q}_{2}$ s. When applied to an internal torsional motion, the transformation is equivalent to the condition, previously stated by Sayvetz [2, 3], that the momentum conjugate to the torsion should be independent of the (remaining) $\dot{q}_{2}$ 's.

An important property of transformations of the type of eq 7 may now be stated. The proof is given in appendix 1 . The coordinates $q_{1}$ may be divided into two or more groups in various ways, and the transformation applied consecutively to each group. The over-all transformations from $q_{1}$ to $q_{1}^{\prime}$ will not, in general, be identical. However, the theorem states that all possible transformations of this type lead to the same reduced matrix $S_{22}^{\prime}$. The importance of this theorem is that in practice it is convenient to apply the transformation consecutively to translation, rotation, and finally, in some cases, to certain internal coordinates.

The Lagrangian equations for the system may now be written as

$$
\begin{gathered}
\dot{p}_{1}^{\prime}=S_{11}^{\prime} \ddot{q}_{1}^{\prime}=0 \\
S_{22}^{\prime} \ddot{q}_{2}+F_{22} q_{2}=0
\end{gathered}
$$

\footnotetext{
${ }^{4}$ Figures in brackets indicate the literature references at the end of this paper.
} 
The first equation shows that the momenta $p_{1}^{\prime}$ are conserved. The second equation may also be written

where

$$
\ddot{q}_{2}+G_{22}^{\prime} F_{22} q_{2}=0,
$$

$$
G_{22}^{\prime}=\left(S_{22}^{\prime}\right)^{-1}
$$

is the reduced reciprocal kinetic-energy matrix for the internal coordinates $q_{2}$. In eq $16, S_{22}^{\prime}$ is defined in terms of the submatrices of $S$. It is proved in appendix 2 that $G_{22}^{\prime}$ may be expressed in terms of the submatrices of $G$, eq 3 , by the equation

$$
G_{22}^{\prime}=\left(S_{22}^{\prime}\right)^{-1}=G_{22}-G_{23} G_{33}^{-1} \widetilde{G}_{23} .
$$

This result has been obtained previously by Wilson [4], although not explicity for the case in which coordinates of the types $q_{1}$ and $q_{3}$ are present simultaneously.

As is well known, eq 18 and 19 lead to the following secular equations for the determination of the normal frequencies of vibration of the coordinates $q_{2}$ :

or,

$$
\left|F_{22}-\lambda S_{22}^{\prime}\right|=0,
$$

$$
\left|G_{22}^{\prime} F_{22}-\lambda E_{22}\right|=0 \text {, }
$$

where $\lambda=4 \pi^{2} \nu^{2}(\nu=$ normal frequency). In the present method, in which $S_{22}^{\prime}$ rather than $G_{22}^{\prime}$ is computed, it is convenient to convert eq 22 to the secular equation of a single matrix, in the form

$$
\left|S_{22}^{\prime} F_{22}^{-1}-\lambda^{-1} E_{22}\right|=0 .
$$

In practice, $F_{22}$ is diagonal, or nearly so, and therefore easily reciprocated. The roots of eq 24 are, of course, the reciprocals of the $\lambda^{\prime}$ s.

It is possible now to state clearly the relative advantages of calculating $S_{22}^{\prime}$ and $G_{22}^{\prime}$ (see eq 3,16 , and 21). In calculating $S_{22}^{\prime}$, the coordinates $q_{3}$ are ignored, but all the elements for $q_{1}$ and $q_{2}$ must be computed. Conversely, in calculating $G_{22}^{\prime}$, the coordinates $q_{1}$ are ignored, but all the elements for $q_{2}$ and $q_{3}$ must be computed. It should also be noted that even with the simplifications described later in this paper, the elements of $S$ are more difficult to compute than those of $G^{5}$ (this state-

\footnotetext{
${ }^{5}$ Wilson [4] has described a now well-known method for the calculation of $G$. A detailed exposition of the method has been given by Meister and Cleveland [5]. A method similar to Wilson's has been developed independently by the Russian workers M. Eliashevich, B. Stepanov, and M. Wolkenstein. References to the papers of these workers are not given here, as we are not directly concerned with the calculation of $G$.
}

ment does not apply to the elements for the external coordinates, but these are just the elements of $G$ that may be ignored). From these considerations it is obvious that the calculation of $G_{22}^{\prime}$ is to be preferred when all or most of the internal coordinates are included in the calculation (so that the number of coordinates of type $q_{3}$ is zero or small). On the other hand, $S_{22}^{\prime}$ is often to be preferred in calculations by the semirigid molecule approximation as the number of coordinates of the type $q_{3}$ is then relatively large, and the number of coordinates remaining in the calculation (type $q_{2}$ ) is relatively small.

If the molecule has the symmetry of one of the point groups, the internal coordinates fall into sets equivalent under the group. The coordinates in an equivalent set may be transformed into each other by the operations (rotations and reflections) of the group. In this case, just as is the case when working with the reciprocal kinetic-energy mtarix $[4,5]$, it is desirable to construct new coordinates, symmetry coordinates, which are linear combinations of equivalent internal coordinates, and which reduce the group. That is, the symmetry coordinates are chosen in such a way that each transforms according to one of the irreducible representations of the group. The advantage of the use of symmetry coordinates is, that there are no interaction elements in the kinetic- or potentialenergy matrices between coordinates which transform according to different irreducible representations. The matrices therefore factor into submatrices (the number of identical submatrices for each representation is equal to the dimension, or degeneracy, of the representation), and the secular equation factors into the corresponding equations of lower degree $[6,7]$.

It will now be shown that the symmetry coordinates may be constructed either before or after applying the transformation of eq 7 , provided that none of the symmetry coordinates involve linear combinations of coordinates of types $q_{1}$ and $q_{2}$. With this restriction the symmetry coordinates may be represented by

$$
\left[\begin{array}{cc}
U_{11} & 0 \\
0 & U_{22}
\end{array}\right]\left[\begin{array}{l}
q_{1} \\
q_{2}
\end{array}\right]
$$

The matrices $U_{11}$ and $U_{22}$ are usually, although not necessarily, orthogonal. The transformed kinetic energy matrix is 


$$
\begin{gathered}
{\left[\begin{array}{cc}
\tilde{U}_{11}^{-1} & 0 \\
0 & \tilde{U}_{22}^{-1}
\end{array}\right]\left[\begin{array}{ll}
S_{11} & S_{12} \\
\tilde{S}_{12} & S_{22}
\end{array}\right]\left[\begin{array}{cc}
U_{11}^{-1} & 0 \\
0 & U_{22}^{-1}
\end{array}\right]} \\
=\left[\begin{array}{cc}
\tilde{U}_{11}^{-1} S_{11} U_{11}^{-1} & \tilde{U}_{11}^{-1} S_{12} U_{22}^{-1} \\
\tilde{U}_{22}^{-1} \tilde{S}_{12} U_{11}^{-1} & \tilde{U}_{22}^{-1} S_{22} U_{22}^{-1}
\end{array}\right]
\end{gathered}
$$

The reduced kinetic-energy matrix is then,

$$
\begin{aligned}
\tilde{U}_{22}^{-1} S_{22} & U_{22}^{-1}- \\
& \tilde{U}_{22}^{-1} \tilde{S}_{12} U_{11}^{-1}\left(\tilde{U}_{11}^{-1} S_{11} U_{11}^{-1}\right)^{-1} \tilde{U}_{11}^{-1} S_{12} U_{22}^{-1} \\
= & \tilde{U}_{22}^{-1} S_{22} U_{22}^{-1}-\tilde{U}_{22}^{-1} \tilde{S}_{12} S_{11}^{-1} S_{12} U_{22}^{-1} \\
= & \tilde{U}_{22}^{-1}\left(S_{22}-\tilde{S}_{12} S_{11}^{-1} S_{12}\right) U_{22}^{-1}
\end{aligned}
$$

which is identical with the result obtained by transforming the original matrix $S_{22}^{\prime}$ of eq 16 by $U_{22}$. It will also be observed that the matrix $S_{22}^{\prime}$ is unaffected by symmetry combinations formed with the coordinates $q_{1}$ (such, for example, as a rotation of axes).

When $U_{22}$ is orthogonal the transformed kineticenergy matrix is $U_{22} S_{22}^{\prime} \tilde{U}_{22}$, but for nonorthogonal $U_{22}$ the general form $\tilde{U}_{22}^{-1} S_{22} U_{22}^{-1}$ must be used. An illustration of the use of a nonorthogonal transformation will occur in the calculations at the end of this paper.

\section{Calculation of the Kinetic-Energy Matrix}

\section{(a) Equations for Generalized Coordinates}

Explicit formulas for the calculation of the elements of the kinetic-energy matrix for generalized internal coordinates are presented in this section. Denote by $m_{i}$ the mass of the $i$ th atom, and by $\mathbf{R}_{i}$ the position vector of the $i$ th atom with respect to a point stationary in an inertial system. Then the kinetic energy of the system of particles is

$$
2 T=\sum_{i} m_{i} \dot{\mathbf{R}}_{i} \cdot \dot{\mathbf{R}}_{i}
$$

where $\dot{\mathbf{R}}_{i}$ is the vector velocity of the $i$ th atom.

It will be assumed here, as in the preceding section, that the positions of the $n$ particles are specified by $3 n$ generalized coordinates $q$, some of which, as before, remain zero for a semirigid molecule. ${ }^{6}$ We may then define a set of vectors,

$$
\mathbf{s}_{m i}=\left(\partial \mathbf{R}_{i} / \partial q_{m}\right) .
$$

${ }^{6}$ In this section specific coordinates will be indicated by letter subscripts, as $q_{m}$ and $q_{n}$.
Thus $\mathbf{s}_{m i}$ is a vector pointing in the direction in which the $i$ th atom moves when $q_{m}$ increases from zero (for the equilibrium configuration) to a small positive value, all other $q$ 's remaining zero, and the magnitude of $\mathbf{s}_{m i}$ is equal to the displacement of the $i$ th atom per unit change in $q_{m}$. Then,

and

$$
\dot{\mathbf{R}}_{i}=\sum_{m} \mathbf{s}_{m i} \dot{q}_{m},
$$

$$
\begin{aligned}
2 T & =\sum_{i} m_{i}\left(\sum_{m} \mathbf{s}_{m i} \dot{q}_{m}\right) \cdot\left(\sum_{n} \mathbf{s}_{n i} \dot{q}_{n}\right) \\
& =\sum_{m, n} \dot{q}_{m} \dot{q}_{n}\left(\sum_{i} m_{i} \mathbf{s}_{m i} \cdot \mathbf{s}_{n i}\right)
\end{aligned}
$$

But from eq 1,

$$
2 T=\sum_{m, n} S_{m n} \dot{q}_{m} \dot{q}_{n}
$$

where $S_{m n}$ is the element in the $m$ th row and $n$th column of the symmetric kinetic-energy matrix $S$. It follows from eq 31 and 32 that

$$
\begin{aligned}
& S_{m m}=\sum_{i} m_{i} \mathbf{s}_{m i} \cdot \mathbf{s}_{m i}, \\
& S_{m n}=S_{n m}=\sum_{i} m_{i} \mathbf{s}_{m i} \cdot \mathbf{s}_{n i}
\end{aligned}
$$

In order to proceed further, define an orthogonal system of $x, y, z$,-axes in the molecule, and denote the unit vectors, directed positively along the axes, by $\mathbf{x}, \mathbf{y}$, and $\mathbf{z}$. Let the system be righthanded, so that $\mathbf{x} \times \mathbf{y}=\mathbf{z}$. The position of the origin of the system, and the directions of the unit vectors, must be uniquely determined with respect to the molecule for any arbitrary (small) displacements of the internal coordinates, but the choice of axes is otherwise arbitrary. In particular, it is not necessary that the origin be at the center of mass of the molecule.

The coordinates of the $i$ th atom are $x_{i}, y_{i}$, and $z_{i}$. For a given choice of axes these coordinates are completely determined when the values of the internal coordinates are given. The position vector of the $i$ th atom with respect to the origin is

$$
\mathbf{r}_{i}=\mathbf{x} x_{i}+\mathbf{y} y_{i}+\mathbf{z} z_{i} .
$$

Three of the generalized coordinates will be taken to be the translations of the origin of the axes in the (instantaneous) directions of the $\mathbf{x}-, \mathbf{y}$-, and $\mathbf{z}$-vectors. The r-vectors of eq 29 are identical for each atom, for each translation, and are simply the unit vectors

$$
\mathbf{s}_{x}=\mathbf{x}, \mathbf{s}_{y}=\mathbf{y}, \mathbf{s}_{z}=\mathbf{z} .
$$


Three more coordinates will be taken to be the three rotations, by angles $\phi_{x} \phi_{y}$ and $\phi_{z}$ about the $x$-, $y$-, and $z$-axes. The $\mathbf{S}$-vectors for these are easily shown to be the vector products,

$$
\mathbf{s}_{\phi x i}=\mathbf{x} \times \mathbf{r}_{i}, \mathbf{s}_{\phi y i}=\mathbf{y} \times \mathbf{r}_{i}, \mathbf{s}_{\phi z i}=\mathbf{z} \times \mathbf{r}_{i} .
$$

For the remaining coordinates, the $(3 n-6)$ internal coordinates,

where,

$$
\mathbf{s}_{m i}=\left(\partial \mathbf{r}_{i} / \partial q_{m}\right)
$$

$$
\left(\partial \mathbf{r}_{i} / \partial q_{m}\right)=\mathbf{x}\left(\partial x_{i} / \partial q_{m}\right)+\mathbf{y}\left(\partial y_{i} / \partial q_{m}\right)+\mathbf{z}\left(\partial z_{i} / \partial q_{m}\right) .
$$

Explicit formulas for the elements of the kinetic-energy matrix may now be obtained from eq 33 and 34, combined with eq 36 to 39 . The reductions of the scalar products of the s-vectors are made by the standard methods of vector analysis. The reduction process of eq 16 may then be applied explicitly to the translations. In this reduction, $S_{11}$ in eq 4 and 16 represents the submatrix for translation, and is a $3 \times 3$ diagonal matrix, with each diagonal element equal to the total mass of the molecule, $M$. $S_{22}$ represents the submatrix for rotations and internal coordinates, and $S_{12}$ the submatrix for the interaction of these coordinates with translation. The elements of $S_{12}$ are simply the $x-, y$-, and $z$-components of linear momentum of the rotations (about the $x, y, z$-axes) and internal coordinates.

The reduced matrix for the rotations and in-

\begin{tabular}{|c|c|c|c|c|c|}
\hline & $\phi_{x}$ & $\phi_{y}$ & $\phi_{z}$ & $q_{m}$ & $q_{n}$ \\
\hline $\begin{array}{l}\phi_{x} \\
\phi_{y} \\
\phi_{z}\end{array}$ & $\begin{aligned} & I_{x} \\
- & -I_{x y} \\
- & I_{z x}\end{aligned}$ & $\begin{array}{l}-I_{x y} \\
I_{y} \\
-I_{y z}\end{array}$ & $\begin{array}{r}-I_{z x} \\
-I_{y z} \\
I_{z}\end{array}$ & $\begin{array}{l}H_{x m} \\
H_{y m} \\
H_{z m}\end{array}$ & $\begin{array}{l}H_{x n} \\
H_{y n} \cdot . \\
H_{z n}\end{array}$ \\
\hline : & . & . & . & . & i \\
\hline$\dot{q_{m}}$ & $\dot{H}_{x m}$ & $\dot{H}_{y m}$ & $\dot{H}_{z m}$ & $\dot{K}_{m m}$ & $\dot{K}_{m n}$ \\
\hline . & : & : & . & : & : \\
\hline$\dot{q_{n}}$ & $\dot{H}_{x n}$ & $\dot{H}_{y n}$ & $\dot{H}_{z n}$ & $\dot{K}_{n m}$ & $\dot{K}_{n n}$ \\
\hline : & · & 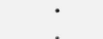 & : & 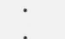 & 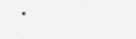 \\
\hline . & . & 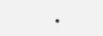 & . & . & 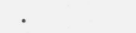 \\
\hline
\end{tabular}
ternal coordinates, corresponding to $S_{22}^{\prime}$ in eq 16 , which is obtained in this way may be written,
The elements for rotation are the moments and products of inertia of the molecule about axes parallel to the $x, y, z$-axes, but passing through the center of mass of the molecule,

$$
\begin{aligned}
I_{x} & =\sum_{i} m_{i}\left(y_{i}^{2}+z_{i}^{2}\right)-M\left(y_{M}^{2}+z_{M}^{2}\right) \\
I_{y} & =\sum_{i} m_{i}\left(z_{i}^{2}+x_{i}^{2}\right)-M\left(z_{M}^{2}+x_{M}^{2}\right) \\
I_{z} & =\sum_{i} m_{i}\left(x_{i}^{2}+y_{i}^{2}\right)-M\left(x_{M}^{2}+y_{M}^{2}\right) \\
I_{x y} & =\sum_{i} m_{i} x_{i} y_{i}-M x_{M} y_{M} \\
I_{y z} & =\sum_{i} m_{i} y_{i} z_{i}-M y_{M} z_{M} \\
I_{z x} & =\sum_{i} m_{i} z_{i} x_{i}-M z_{M} x_{M}
\end{aligned}
$$

The total mass of the molecule has been denoted by $M$, and the coordinates of the center of mass of the molecule, for the equilibrium configuration, by $x_{M}, y_{M}$, and $z_{M}$. The first term in each equation of 41 is the moment or product of inertia about the arbitrary origin; the second term arises from the reduction process of eq 16, and vanishes if the origin coincides with the (equilibrium) position of the center of mass.

The elements for the interaction of rotation and internal coordinates may be written

$$
H_{x m}=\mathbf{x} \cdot \mathbf{H}_{m}, H_{y m}=\mathbf{y} \cdot \mathbf{H}_{m}, H_{z m}=\mathbf{z} \cdot \mathbf{H}_{m},
$$

where $\mathbf{H}_{m}$ is the vector

$$
\mathbf{H}_{m}=\sum_{i} m_{i} \mathbf{r}_{i} \times\left(\partial \mathbf{r}_{i} / \partial q_{m}\right)-\mathbf{r}_{M} \times \sum_{i} m_{i}\left(\partial \mathbf{r}_{i} / \partial q_{m}\right)
$$

The vector from the origin of the axes to the center of mass of the molecule has been represented by $\mathbf{r}_{M}$,

$$
\mathbf{r}_{M}=M^{-1} \sum_{i} m_{i} \mathbf{r}_{i}=\mathbf{x} x_{M}+\mathbf{y} y_{M}+\mathbf{z} z_{M}
$$


If the origin of the $x, y, z$-axes coincides with the (equilibrium) position of the center of mass of the molecule, the vector $\mathbf{r}_{M}$, and the second term in eq 43, vanish. The second term arises from the reduction process of eq 16 . The first term in eq 43 represents the vector angular momentum of the motion $q_{m}$ about the origin, while $\mathbf{H}_{m}$ represents the vector angular momentum of $q_{m}$ about the (equilibrium) center of mass. The quantities $H_{x m}, H_{y m}$, and $H_{z m}$ are therefore the components of angular momentum of $q_{m}$ about axes through the center of mass parallel to the $x, y$, z-axes.

For internal coordinates,

$K_{m m}=\sum_{i} m_{i}\left(\partial \mathbf{r}_{i} / \partial q_{m}\right)^{2}-M^{-1}\left[\sum_{i} m_{i}\left(\partial \mathbf{r}_{i} / \partial q_{m}\right)\right]^{2}$

and for the interaction of two internal coordinates,

$$
\begin{aligned}
K_{m n} & =K_{n m} \sum_{i} m_{i}\left(\partial \mathbf{r}_{i} / \partial q_{m}\right) \cdot\left(\partial \mathbf{r}_{i} / \partial q_{n}\right) \\
& -M^{-1}\left[\sum_{i} m_{i}\left(\partial \mathbf{r}_{i} / \partial q_{m}\right)\right] \cdot\left[\sum_{i} m_{i}\left(\partial \mathbf{r}_{i} / \partial q_{n}\right)\right]
\end{aligned}
$$

The second term in eq 45 may also be written $M\left(\partial \mathbf{r}_{M} / \partial q_{m}\right)^{2}$, and therefore vanishes if the position of the center of mass of the molecule is independent of $q_{m}$. Similarly, the second term in eq 46 is $M\left(\partial \mathbf{r}_{M} / \partial q_{m}\right) \cdot\left(\partial \mathbf{r}_{M} / \partial q_{n}\right)$, and vanishes if the center of mass is independent of either $g_{m}$ or $q_{n}$ (or both). However, the vanishing of the (equilibrium) vector $\mathbf{r}_{M}$ is not, of itself, sufficient to cause these terms to vanish. The second terms in eq 45 and 46 represent the kinetic energy of translation of the center of mass of the molecule, and arise in the reduction process of eq 16 . The first terms in these equations represent the kinetic energy of the internal coordinates computed with respect to the arbitrary origin. Subtraction of the second term from the first yields $K_{m m}$ and $K_{m n}$, which represent the kinetic energy of the internal coordinates computed with respect to the instantaneous position of the center of mass.

The set of atoms associated with the internal coordinate $q_{m}$ will be defined to include those atoms which move with respect to the $x, y, z$-axes when the coordinate $g_{m}$ varies. This set is not to be regarded as fundamental, because the atoms included in it depend, in general, on the definition of the axes. The vector $\left(\partial \mathbf{r}_{i} / \partial q_{m}\right)$ vanishes by definition for all atoms not in the set associated with $q_{m}$. As a result, the summations in eq 43 and 45 need extend only over the set of atoms assoeiated with $q_{m}$. In eq 46 the two summations in brackets extend over the sets of atoms associated with $q_{m}$ and $q_{n}$, respectively, whereas the first summation in eq 46 extends only over those atoms included in both of these sets.

The final reduced kinetic-energy matrix is obtained from the matrix 40 by applying the reduction process of eq 16 to the rotations, and to any internal coordinates that do not appear in the potential-energy expression. The elements of the matrix of eq 40 are not independent, in general, of the choice of the $x, y, z$-axes, but the reduced matrix is independent of the axes after the overall rotations have been removed. The reduced matrix is most easily obtained numerically, by applying the reduction process of eq 10 to one row and column of the matrix at a time (so that in each step $S_{11}$ in eq 16 is a matrix with a single element). This procedure makes it unnecessary to reciprocate directly the submatrix for the coordinates that are to be removed.

\section{(b) Equations for semirigid molecules}

The equations for the calculation of the kineticenergy matrix for generalized internal coordinates have been given in the preceding section. These equations may now be applied to the two types of internal coordinates which will be found useful in specifying the configuration of a semirigid molecule. These are coordinates that represent either a translation or a rotation of their associated sets of atoms with respect to the $x, y, z$-axes. Internal translational and rotational coordinates will be represented by $q_{t}$ and $q_{r}$, respectively, where $q_{t}$ is the distance of translation, and $q_{r}$ is the angle of rotation, both measured from the equilibrium configuration.

Let $\bar{\mu}$ be a unit vector in the direction of translation for the coordinate $q_{t}$, with components $\mu_{x}, \mu_{y}$, and $\mu_{z}$, and let $\bar{\xi}$ be a unit vector directed along the axis of rotation for the coordinate $q_{r}$, with components $\xi_{x}, \xi_{y}$, and $\xi_{z}$. Let $\bar{\rho}_{\imath}$ be the position vector of the $i$ th atom associated with $q_{r}$, with respect to an arbitrary point on the axis of rotation for $q_{r}$. In terms of these vectors eq 38 becomes,

and

$$
\left(\partial \mathbf{r}_{i} / \partial q_{t}\right)=\bar{\mu}
$$

$$
\left(\partial \mathbf{r}_{i} / \partial q_{r}\right)=\bar{\xi} \times \bar{\rho}_{i},
$$

for translational and rotational coordinates, respectively. Equations 47 and 48 may now be substituted in eq 42 to 46 , to obtain the elements 
of the kinetic-energy matrix, eq 40, in vector form. These vector expressions may then be reduced by the usual methods to more useful scalar expressions involving the masses, moments of mass, and moments of inertia of the associated sets of atoms. The details of these reductions will be omitted and only the final formulas stated.

Some additional definitions will be necessary. The total mass of the set of atoms associated with a coordinate $q_{m}(m=t$ or $r)$ will be denoted by $M_{m}$, and the coordinates of the center of mass of the set of atoms by $x_{M m}, y_{M m}$, and $z_{M m}$. The corresponding quantities for the entire molecule are $M$, and $x_{M}, y_{M}$, and $z_{M}$.

For each rotational coordinate, $q_{r}$, define a set of orthogonal right-handed $\xi, \eta, \zeta$-axes, with the origin at a point $x_{o r}, y_{o r}, z_{o r}$, on the axis of rotation, but otherwise aribtrary, and the $\xi$-axis coinciding with the axis of rotation. The direction cosines of the $\xi, \eta, \zeta$-axes with respect to the $x, y, z$-axes, written in matrix form, are

$$
\left[\begin{array}{ccc}
\xi_{x} & \eta_{x} & \zeta_{x} \\
\xi_{y} & \eta_{y} & \zeta_{y} \\
\xi_{z} & \eta_{z} & \zeta_{z}
\end{array}\right]
$$

The determinant of this matrix will be +1 if both the $x, y, z$-, and $\xi, \eta, \zeta$-axes are right-handed.

The following moments and products of inertia of the set of atoms associated with $q_{r}$ will be required,

$$
\left.\begin{array}{rl}
I_{\xi} & =\sum_{i} m_{i}\left(\eta_{i}^{2}+\zeta_{i}^{2}\right) \\
I_{\xi \eta} & =\sum_{i} m_{i} \xi_{i} \eta_{i} \\
I_{\zeta \xi} & =\sum_{i} m_{i} \zeta_{i} \xi_{i}
\end{array}\right\}
$$

$I_{\xi}$ is the moment of inertia about the axis of rotation. The products of inertia, $I_{\xi \eta}$ and $I_{\zeta \xi}$, vanish if (1) the $\xi$-axis is a twofold, or higher, axis of symmetry for the associated set of atoms; (2) the $\eta, \zeta$-plane is a plane of symmetry; or (3) if a threefold, or higher, axis of symmetry lies in the $\eta$, $\zeta$-plane, and intersects the $\xi$-axis. $I_{\xi \eta}$ (but not necessarily $I_{\zeta \xi}$ ) vanishes if (1) the $\zeta, \xi$-plane is a plane of symmetry or (2) the $\eta$-axis is a twofold axis of symmetry.

Let $d_{r}$ be the perpendicular distance from the center of mass of the set of atoms associated with $q_{r}$ to the axis of rotation, or $\xi$-axis, and let $\lambda_{x}, \lambda_{y}$, and $\lambda_{z}$ be the direction cosines, with respect to the $x, y, z$-axes, if the line along which this center of mass moves when $q_{r}$ increases from zero to a small positive value.

That is,

$$
d_{r}=\left(\eta_{M}^{2}+\zeta_{M}^{2}\right)
$$

and

$$
\left.\begin{array}{l}
\lambda_{x}=\left(\eta_{M r} \zeta_{x}-\zeta_{M r} \eta_{x}\right) / d_{r} \\
\lambda_{y}=\left(\eta_{M r} \zeta_{y}-\zeta_{M r} \eta_{y}\right) / d_{r} \\
\lambda_{z}=\left(\eta_{M r} \zeta_{z}-\zeta_{M r} \eta_{z}\right) / d_{r}
\end{array}\right\}
$$

where,

$$
\eta_{M r}=M_{r}^{-1} \sum_{i} m_{i} \eta_{i}, \zeta_{M r}=M_{r}^{-1} \sum_{i} m_{i} \zeta_{i} .
$$

The sums in eq 50 and 53 extend over the atoms associated with $q_{r}$, Atoms on the axis of rotation are not associated with $q_{r}$, according to the definition given in the preceding section. It may be more convenient to calculate these quantities as follows. Define $\alpha_{x}, \alpha_{y}$, and $\alpha_{z}$ to be equal to their respective cofactors in the determinant,

$$
\left|\begin{array}{ccc}
\alpha_{x} & \alpha_{y} & \alpha_{z} \\
\xi_{x} & \xi_{y} & \xi_{z} \\
\left(x_{M \tau}-x_{o r}\right) & \left(y_{M r}-y_{o r}\right) & \left(z_{M \tau}-z_{o r}\right)
\end{array}\right|
$$

Then

$$
d_{r}=\left(\alpha_{x}^{2}+\alpha_{y}^{2}+\alpha_{z}^{2}\right),
$$

and

$$
\lambda_{x}=\left(\alpha_{x} / d_{r}\right), \lambda_{y}=\left(\alpha_{y} / d_{r}\right), \lambda_{z}=\left(\alpha_{z} / d_{r}\right) .
$$

The equations for the elements of the kineticenergy matrix, eq 40, may now be stated. For a translational coordinate, $q_{t}, H_{x t}, H_{y t}$, and $H_{z t}$ are equal to their respective cofactors in the determinant

$$
\left|\begin{array}{crc}
H_{x t} & H_{y t} & H_{z t} \\
M_{t} \mu_{x} & M_{t \mu_{y}} & M_{t \mu_{z}} \\
\left(x_{M t}-x_{M}\right) & \left(y_{M t}-y_{M}\right) & \left(z_{M t}-z_{M}\right)
\end{array}\right|
$$


If the translation arises from the stretching of a bond, $\mu_{x}, \mu_{y}$, and $\mu_{z}$ are the direction cosines of the bond.

For a rotational coordinate, $q_{r}$,

$$
\left.\begin{array}{l}
H_{x r}=H_{x r}^{(1)}+H_{x r}^{(2)} \\
H_{y r}=H_{y r}^{(1)}+H_{y r}^{(2)} \\
H_{z r}=H_{z r}^{(1)}+H_{z r}^{(2)}
\end{array}\right\},
$$

where $H_{x r}^{(1)}, H_{y r}^{(1)}$, and $H_{z r}^{(1)}$ are defined by the equations

$$
\begin{aligned}
& H_{x r}^{(1)}=\xi_{x} I_{\xi}-\eta_{x} I_{\xi \eta}-\zeta_{x} I_{\zeta \xi} \\
& H_{y r}^{(1)}=\xi_{y} I_{\xi}-\eta_{y} I_{\xi \eta}-\zeta_{y} I_{\zeta \xi} \\
& H_{z r}^{(1)}=\xi_{z} I_{\xi}-\eta_{z} I_{\xi \eta}-\zeta_{z} I_{\zeta \xi}
\end{aligned},,
$$

and $H_{x r}^{(2)}, H_{y r}^{(2)}$, and $H_{z r}^{(2)}$ are equal to their respective cofactors in the determinant

$$
\left|\begin{array}{ccc}
H_{x r}^{(2)} & H_{y r}^{(2)} & H_{z r}^{(2)} \\
M_{r} d_{r} \lambda_{x} & M_{r} d_{r} \lambda_{y} & M_{r} d_{r} \lambda_{z} \\
\left(x_{o r}-x_{M}\right) & \left(y_{o r}-y_{M}\right) & \left(z_{o r}-z_{M}\right)
\end{array}\right| .
$$

In many cases, $I_{\xi \eta}=I_{\zeta \xi}=0$, and eq 59 reduces to

$$
H_{x r}^{(1)}=\xi_{x} I_{\xi}, H_{y r}^{(1)}=\xi_{y} I_{\xi}, H_{z r}^{(1)}=\xi_{2} I_{\xi} .
$$

Before stating the formulas for the remaining elements, $K_{m m}$, and $K_{m n}$, of eq 40 , it will be necessary to differentiate between connected and unconnected coordinates. If the sets of atoms associated with two coordinates $q_{m}$ and $q_{n}$ contain no atoms in common, the two coordinates will be said to be unconnected-otherwise they are connected. Whether two coordinates are connected or unconnected will be dependent, in general, on the way in which the $x, y, z$-axes are defined. The only types of connected coordinates that will be considered are those for which the set of atoms for one coordinate, say $q_{n}$, is completely contained within the set of atoms for the other coordinate, $q_{m}$. A special case is that in which the sets of atoms for $q_{m}$ and $q_{n}$ are identical. If $q_{m}$ and $q_{n}$ are unconnected, this fact will be indicated explicitly by writing $K_{m n}\left(=K_{n m}\right)$ as $K_{m, n}\left(=K_{n, m}\right)$. If $q_{m}$ and $q_{n}$ are connected, and the set of atoms for $q_{n}$ is completely contained within the set of atoms for $q_{m}$, then $K_{m n}\left(=K_{n m}\right)$ will be written $K_{m(n)}\left(=K_{(n) m}\right)$. If the set of atoms for $q_{m}$ and $q_{n}$ are identical, $K_{m(n)}=K_{(m) n}$. Subscripts $t$ and $r$ will again be used to indicate translational and rotational coordinates, and a second coordinate of a given type will be distinguished by the use of primes. The equations for the several possible forms of $K_{m m}$ and $K_{m n}$ may now be written as

$K_{t t}=M_{t}\left(1-M^{-1} M_{t}\right)$

$K_{t, \ell^{\prime}}=-M^{-1} M_{t} M_{t}^{\prime}\left(\mu_{x} \mu^{\prime}{ }_{x}+\mu_{y} \mu^{\prime}{ }_{y}+\mu_{z} \mu_{z}^{\prime}\right)$

$K_{t\left(t^{\prime}\right)}=M_{t}^{\prime}\left(1-M^{-1} M_{t}\right)\left(\mu_{x} \mu^{\prime}{ }_{x}+\mu_{j} \mu^{\prime}{ }_{y}+\mu_{z} \mu_{z}^{\prime}\right)$

$K_{t, r}=-M^{-1} M_{t} M_{r} \Delta_{t r}$

$K_{t(r)}=M_{r}\left(1-M^{-1} M_{t}\right) \Delta_{t r}$

$K_{r(t)}=M_{t} \Delta_{r(t)}-M^{-1} M_{t} M_{r} \Delta_{t r}$

$K_{r r}=I_{\xi}-M^{-1} M_{r}^{2} d_{r}^{2}$

$K_{r, r^{\prime}}=-M^{-1} M_{r} M^{\prime}{ }_{r} d_{r} d^{\prime}{ }_{r}\left(\lambda_{x} \lambda^{\prime}{ }_{x}+\lambda_{y} \lambda^{\prime}{ }_{y}+\lambda_{z} \lambda_{z}^{\prime}\right)$

$K_{r\left(r^{\prime}\right)}=\left(\xi_{x} H_{x r}^{(1) \prime}+\xi_{y} H_{y r}^{(1) \prime}+\xi_{z} H_{z r}^{(1) \prime}\right)+M_{r}^{\prime} d_{r}^{\prime} \Delta_{r\left(r^{\prime}\right)}$ $-M^{-1} M_{r} M_{r}^{\prime} d_{r} d_{r}^{\prime}\left(\lambda_{x} \lambda_{x}^{\prime}+\lambda_{y} \lambda_{y}^{\prime}+\lambda_{z} \lambda_{z}^{\prime}\right)$

The symbols $\Delta_{t r}, \Delta_{r(t)}$, and $\Delta_{r\left(r^{\prime}\right)}$, represent the following determinants:

$$
\begin{aligned}
\Delta_{t r} & =d_{r}\left(\mu_{x} \lambda_{x}+\mu_{y} \lambda_{y}+\mu_{z} \lambda_{z}\right) \\
& =\left|\begin{array}{ccc}
\mu_{x} & \mu_{y} & \mu_{z} \\
\xi_{x} & \xi_{y} & \xi_{z} \\
\left(x_{M r}-x_{o r}\right) & \left(y_{M r}-y_{o r}\right) & \left(z_{M \tau}-z_{o r}\right) \\
\mu_{x} & \mu_{y} & \mu_{z} \\
\xi_{x} & \xi_{y} & \xi_{z} \\
\left(x_{M t}-x_{o r}\right) & \left(y_{M t}-y_{o r}\right) & \left(z_{M t}-z_{o r}\right)
\end{array}\right|, \\
\Delta_{r\left(r^{\prime}\right)} & =\left|\begin{array}{ccc}
\lambda_{x}^{\prime} & \lambda_{y}^{\prime} & \lambda_{z}^{\prime} \\
\xi_{x} & \xi_{y} & \xi_{z} \\
\left(x_{o \tau}^{\prime}-x_{o r}\right) & \left(y_{o r}^{\prime}-y_{o r}\right) & \left(z_{o r}^{\prime}-z_{o r}\right)
\end{array}\right|
\end{aligned}
$$

In eq $70, H_{x r}^{(1) \prime}, H_{y r}^{(1) \prime}$, and $H_{z r}^{(1)}$, are the quantities calculated from eq 59 (with $r=r^{\prime}$ ). When eq 61 applies, the first term in parentheses in eq 70 reduces to,

$$
\left(\xi_{x} \xi_{x}^{\prime}+\xi_{y} \xi_{y}^{\prime}+\xi_{z} \xi_{z}^{\prime}\right) I_{\xi}^{\prime}
$$

If the center of mass of the set of atoms associated with a rotation, $q_{r}$, lies on the axis of rotation, so that $d_{r}=0, q_{r}$ will be called a balanced 
rotation. For a balanced rotation, $H_{x r}^{(2)}=H_{y r}^{(2)}=$ $H_{2 r}^{(2)}=0$, in eq 58 , and there are obvious simplifications in eq 65 to 70 , as $\Delta_{t r}=0$ (in addition to $\left.d_{r}=0\right)$. A balanced rotation may be called accidentally balanced if the $\xi$-axis is not an axis of symmetry of the associated set of atoms. Such cases are very unlikely to occur. As a rule, the $\xi$-axis for a balanced rotation will be a twofold or higher axis of symmetry. In this case, $I_{\xi \eta}=I \xi_{\xi}=0$, and eq 59 is replaced by eq 61 . However, it is not necessary that a rotation be balanced for $I_{\xi \eta}$ and $I_{\zeta \xi}$ to vanish.

\section{Application to Propane, Toluene, and Ethylbenzene}

\section{Kinetic-Energy Matrices}

The application of this method to certain vibrations in ethylbenzene will be described in this section. In order to obtain the necessary force constants for ethylbenzene, it also has been necessary to set up the kinetic-energy matrices for propane and toluene. In the semirigid model of ethylbenzene for which the calculation has been carried out, it is assumed that the benzene ring and the attached hydrogens move as a rigid body, and also that the methylene and methyl groups move as rigid bodies. That is, the force constants for all deformations of the benzene ring and attached hydrogens and for deformation of the $\mathrm{H}-\mathrm{C}-\mathrm{H}$ angles in the methylene and methyl groups have been set equal to infinity. The force constants for the stretching of the two $\mathrm{C}-\mathrm{C}$ bonds in the ethyl group have also been assumed infinite. ${ }^{7}$ In order that the force constants obtained from the observed frequencies of propane and toluene should be on a comparable basis, corresponding assumptions have been made for these molecules. The coordinates remaining in the problem are therefore the rocking and twisting motions of these rigid groups.

The total number of internal coordinators in ethylbenzene is 48; of these, 8 are retained in the present calculation, and 2 are assumed to have zero frequency. In calculating the kinetic-energy matrix by the present method it is necessary to consider only these 10 internal coordinates, plus the

\footnotetext{
${ }^{7}$ As no bond stretchings are included in the present calculations, they will not illustrate the use of the formulas for translational internal coordinates given in section II, $2 \mathrm{~b}$.
}

3 over-all rotations, and then to remove the 5 coordinates of zero frequency, as discussed in section II,1. In order to obtain the reciprocal kinetic-energy matrix for the 8 internal coordinates, it would be necessary to set the matrix up for the 46 internal coordinates of nonzero frequency, and then to remove 38 coordinates, as discussed in section II,1.

Before enumerating the coordinates, the symmetry properties of the molecules will be reviewed briefly. The propane molecule has the symmetry $C_{2 v}$, with the nondegenerate representations $A_{1}$, $A_{2}, B_{1}$, and $B_{2}$. Representations $A$ and $B$ are symmetric and antisymmetric, respectively, to rotation by 180 degrees about the twofold axis. Representations with subscripts 1 and 2 are symmetric and antisymmetric, respectively, to reflection in the symmetry plane. The toluene and ethylbenzene molecules have the symmetry $C_{1 h}$, with nondegenerate representations $A^{\prime}$ and $A^{\prime \prime}$, which are symmetric and antisymmetric, respectively, to reflection in a plane perpendicular to the plane of the benzene ring. ${ }^{8}$

The notation adopted for the internal coordinates is as follows. In-plane and out-of-plane (with respect to the symmetry plane) rocking motions are denoted by $\sigma_{i}$ and $\pi_{i}$, respectively. Internal rotational motions are denoted by $\phi_{i}$. The subscript $i$ indicates the group, as follows: Propane, methyl (1 and 2), and methylene (3); ethylbenzene, methyl (1), ethyl (2), and methylene (3); and toluene, methyl (1), and methyl group (2). ${ }^{9} \quad$ On this basis methylene twisting is represented by $\phi_{3}$. The $\mathrm{C}-\mathrm{C}-\mathrm{C}$ angle bending (in propane and ethylbenzene) is denoted by $\alpha$. The coordinates represent in each case the angular deformation from the equilibrium configuration. In addition the over-all, rotations about the $x, y, z$-axes will be denoted by $\phi_{x}, \phi_{y}$, and $\phi_{z}$, respectively.

\footnotetext{
${ }^{8}$ The equilibrium configuration assumed for the ethylbenzene moleclue is that in which the plane of the benzene ring is perpendicular to the plane of the $\mathrm{C}-\mathrm{C}-\mathrm{C}$ angle in the ethyl group. The angle of rotation of the methyl groups in these molecules does not affect the quantities entering into the calculations.

${ }^{9}$ The axes for methyl and methylene rocking pass through the carbon atoms of the $\mathrm{CH}_{3}$ and $\mathrm{CH}_{2}$ groups, respectively, so that these are motions of the hydrogen atoms only. The axis for ethyl rocking passes through the phenyl carbon atom, se that the entire $\mathrm{C}_{2} \mathrm{H}_{\varepsilon}$ group moves as a unit. The motion in toluene of the entire $\mathrm{CH}_{3}$ group as a unit about an axis through the phenyl carbon atom has been called "methyl group" rocking to distinguish it from methyl rocking. Methyl rocking may conveniently be denoted by $\mathrm{CH}_{3}$ rocking, and methyl group rocking by $-\mathrm{CH}_{3}$ rocking.
} 
In the case of propane it is necessary to construct the following symmetry coordinates:

$$
\left.\begin{array}{c}
\sigma^{+}=(1 / \sqrt{2})\left(\sigma_{1}+\sigma_{2}\right) \\
\sigma^{-}=(1 / \sqrt{2})\left(\sigma_{1}-\sigma_{2}\right) \\
\pi^{+}=(1 / \sqrt{2})\left(\pi_{1}+\pi_{2}\right) \\
\pi^{-}=(1 / \sqrt{2})\left(\pi_{1}-\pi_{2}\right) \\
\phi^{+}=(1 / \sqrt{2})\left(\phi_{1}+\phi_{2}\right) \\
\phi^{-}=(1 / \sqrt{2})\left(\phi_{1}-\phi_{2}\right)
\end{array}\right\}
$$

These transformations are orthogonal, with the matrix,

$$
(1 / \sqrt{2})\left[\begin{array}{rr}
1 & 1 \\
1 & -1
\end{array}\right] \text {. }
$$

From eq 26 there are obtained the relations

$$
\left.\begin{array}{rl}
\left(\sigma^{+}, \sigma^{+}\right) & =(1 / 2)\left[\left(\sigma_{1}, \sigma_{1}\right)+\left(\sigma_{2}, \sigma_{2}\right)\right]+\left(\sigma_{1}, \sigma_{2}\right) \\
\left(\sigma^{-}, \sigma^{-}\right) & =(1 / 2)\left[\left(\sigma_{1}, \sigma_{1}\right)+\left(\sigma_{2}, \sigma_{2}\right)\right]-\left(\sigma_{1}, \sigma_{2}\right) \\
\left(\sigma^{+}, \sigma^{-}\right) & =(1 / 2)\left[\left(\sigma_{1}, \sigma_{1}\right)-\left(\sigma_{2}, \sigma_{2}\right)\right]=0 \\
\left(\sigma^{+}, q\right) & =(1 / \sqrt{2})\left[\left(\sigma_{1}, q\right)+\left(\sigma_{2}, q\right)\right] \\
\left(\sigma^{-}, q\right) & =(1 / \sqrt{2})\left[\left(\sigma_{1}, q\right)-\left(\sigma_{2}, q\right)\right]
\end{array}\right\}
$$

where $\left(\sigma^{+}, \sigma^{+}\right)$is the diagonal element in the kinetic-energy matrix for $\sigma^{+},\left(\sigma^{+}, \sigma^{-}\right)$is the element connecting $\sigma^{+}$and $\sigma^{-}$(equal to $\left(\sigma^{-}, \sigma^{+}\right)$), etc. $q$ represents any coordinate other than $\sigma_{1}, \sigma_{2}, \sigma^{+}$, or $\sigma^{-}$. Similar relations hold for the $\pi$ and $\phi$ co-

\begin{tabular}{|c|c|c|}
\hline Symmetry & Coordinate $^{a}$ & Description \\
\hline \multicolumn{3}{|c|}{ PROPANE $\left(\mathrm{C}_{2 \mathrm{\sigma}}\right)$} \\
\hline$A_{1}$ & $\begin{array}{l}\sigma^{-} \\
\alpha\end{array}$ & $\begin{array}{l}\mathrm{CH}_{3} \text { rocking } \\
\mathrm{C}-\mathrm{C}-\mathrm{C} \text { bending }\end{array}$ \\
\hline \multirow{2}{*}{$A_{2}$} & $\begin{array}{l}\varphi_{z} \\
\varphi^{+}\end{array}$ & $\begin{array}{l}\text { Over-all rotation } \\
\mathrm{CH}_{3} \text { rotation }\end{array}$ \\
\hline & $\begin{array}{l}\varphi_{3} \\
\pi^{+}\end{array}$ & $\begin{array}{l}\mathrm{CH}_{2} \text { twisting } \\
\mathrm{CH}_{3} \text { rocking }\end{array}$ \\
\hline \multirow{2}{*}{$B_{1}$} & $\varphi_{y}$ & Over-all rotation \\
\hline & $\begin{array}{l}\sigma^{+} \\
\sigma_{3}\end{array}$ & $\begin{array}{l}\mathrm{CH}_{3} \text { rocking } \\
\mathrm{CH}_{2} \text { rocking }\end{array}$ \\
\hline \multirow{2}{*}{$B_{2}$} & $\begin{array}{l}\varphi_{x} \\
\varphi^{-}\end{array}$ & $\begin{array}{l}\text { Over-all rotation } \\
\mathrm{CH}_{3} \text { rotation }\end{array}$ \\
\hline & $\begin{array}{l}\pi^{-} \\
\pi_{3}\end{array}$ & $\begin{array}{l}\mathrm{CH}_{3} \text { rocking } \\
\mathrm{CH}_{2} \text { rocking }\end{array}$ \\
\hline \multicolumn{3}{|c|}{ ETHYLBENZENE $\left(\mathrm{C}_{1 h}\right)$} \\
\hline \multirow[b]{2}{*}{$A^{\prime}$} & $\varphi_{y}$ & Over-all rotation \\
\hline & $\begin{array}{l}\sigma_{1} \\
\sigma_{2} \\
\sigma_{3} \\
\alpha\end{array}$ & $\begin{array}{l}\mathrm{CH}_{3} \text { rocking } \\
\mathrm{C}_{2} \mathrm{H}_{5} \text { rocking } \\
\mathrm{CH}_{2} \text { rocking } \\
\mathrm{C}-\mathrm{C}-\mathrm{C} \text { bending }\end{array}$ \\
\hline \multirow{2}{*}{$A^{\prime \prime}$} & $\begin{array}{l}\varphi_{x} \\
\varphi_{z} \\
\varphi_{1} \\
\varphi_{2}\end{array}$ & $\begin{array}{l}\text { Over-all rotation } \\
\text { Over-all rotation } \\
\mathrm{CH}_{3} \text { rotation } \\
\mathrm{C}_{2} \mathrm{H}_{5} \text { rotation }\end{array}$ \\
\hline & $\begin{array}{l}\varphi_{3} \\
\pi_{1} \\
\pi_{2} \\
\pi_{3}\end{array}$ & $\begin{array}{l}\mathrm{CH}_{2} \text { twisting } \\
\mathrm{CH}_{3} \text { rocking } \\
\mathrm{C}_{2} \mathrm{H}_{5} \text { rocking } \\
\mathrm{CH}_{2} \text { rocking }\end{array}$ \\
\hline \multicolumn{3}{|c|}{ TOLUENE $\left(\mathrm{C}_{1 h}\right)$} \\
\hline \multirow{2}{*}{$A^{\prime}$} & $\varphi_{y}$ & Over-all rotation \\
\hline & $\begin{array}{l}\sigma_{1} \\
\sigma_{2}\end{array}$ & $\begin{array}{l}\mathrm{CH}_{3} \text { rocking } \\
-\mathrm{CH}_{3} \text { rocking } 9\end{array}$ \\
\hline \multirow{2}{*}{$A^{\prime \prime}$} & $\begin{array}{l}\varphi_{x} \\
\varphi_{z} \\
\varphi_{1}\end{array}$ & $\begin{array}{l}\text { Over-all rotation } \\
\text { Over-all rotation } \\
\mathrm{CH}_{3} \text { rotation }\end{array}$ \\
\hline & $\begin{array}{l}\pi_{1} \\
\pi_{2}\end{array}$ & $\begin{array}{l}\mathrm{CH}_{3} \text { rocking } \\
-\mathrm{CH}_{3} \text { rocking }{ }^{9}\end{array}$ \\
\hline
\end{tabular}
ordinates. The elements for the internal coordinates are first calculated from the equations given in section II, $2 \mathrm{~b}$, and the elements for the symmetry coordinates are then calculated from eq 76 .

The notation used for the coordinates, their symmetries or representations, and their conventional descriptions are summarized in table 1 . Interaction terms in the kinetic- and potentialenergy matrices between coordinates belonging to different representations vanish identically.
TABLE 1.-Notation and symmetry for coordinates

${ }_{a}$ The coordinates above the dashed line in each representation were removed from the kinetic-energy matrices (see text). 
The dimensions used in the calculations are as follows. The benzene ring was assumed to be a plane hexagon, with the length of the $\mathrm{C}-\mathrm{C}$ bonds 1.39 angstrom units. The other bond lengths, in angstrom units, were 1.54 for the $\mathrm{C}-\mathrm{C}$ bonds in propane and in the alkyl groups (including the bond joining the alkyl group to the ring [8]), 1.09 for the $\mathrm{C}-\mathrm{H}$ bonds in propane and the alkyl groups, and 1.08 for the $\mathrm{C}-\mathrm{H}$ bonds on the benzene ring. Tetrahedral angles were assumed in propane and in the alkyl groups. In every case the numerical values of the elements of the kinetic-energy matrices (tables 2 and 3 ) have the units of mass in atomic-weight units multiplied by the square of a length in angstrom units.

TABLE 2.-Kinetic-energy matrices for propane before removal of over-all and internal rotations
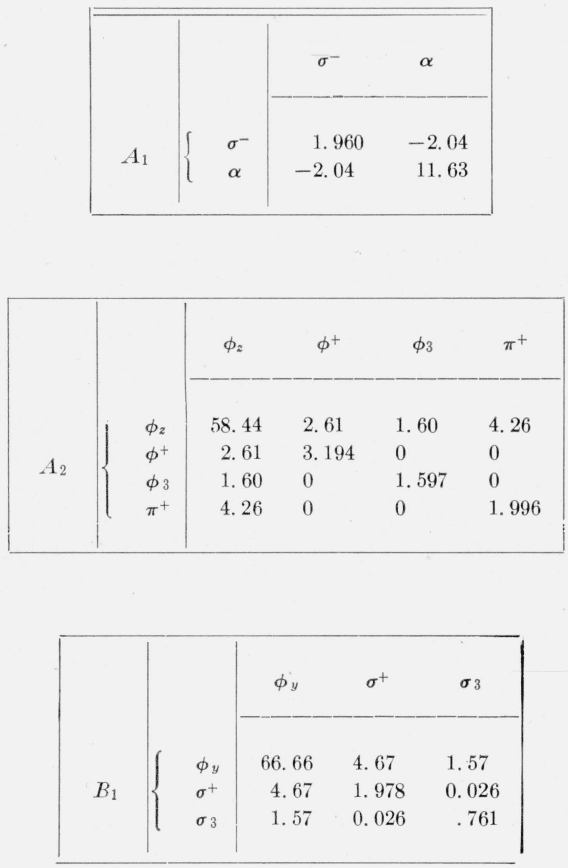

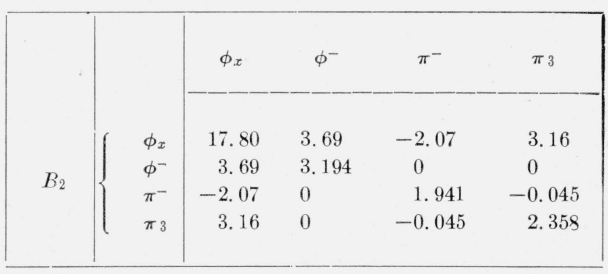

TARLE 3.-Kinetic-energy matrices for propane, toluene, and ethylbenzene

\begin{tabular}{|c|c|c|c|}
\hline \multicolumn{4}{|c|}{ Propane $\left(\mathrm{C}_{2 v}\right)$} \\
\hline & & $\sigma^{-}$ & $\alpha$ \\
\hline$A_{1}$ & $\begin{array}{l}\sigma^{-} \\
\alpha\end{array}$ & $\begin{array}{r}1.96 \\
-2.04\end{array}$ & $\begin{array}{c}-2.04 \\
11.6\end{array}$ \\
\hline & & $\phi_{3}$ & $\pi^{+}$ \\
\hline$A_{2}$ & $\begin{array}{l}\phi_{3} \\
\pi^{+}\end{array}$ & $\begin{array}{c}1.55 \\
-0.120\end{array}$ & $\begin{array}{c}-0.120 \\
1.67\end{array}$ \\
\hline & & $\sigma^{+}$ & $\sigma_{3}$ \\
\hline$B_{1}$ & $\begin{array}{l}\sigma^{+} \\
\sigma_{3}\end{array}$ & $\begin{array}{c}1.65 \\
-0.084\end{array}$ & $\begin{array}{r}-0.084 \\
.724\end{array}$ \\
\hline & & $\pi^{-}$ & $\pi_{3}$ \\
\hline$B_{2}$ & $\begin{array}{l}\pi^{-} \\
\pi_{3}\end{array}$ & $\begin{array}{l}1.63 \\
0.44\end{array}$ & $\begin{array}{l}0.44 \\
1.62\end{array}$ \\
\hline
\end{tabular}

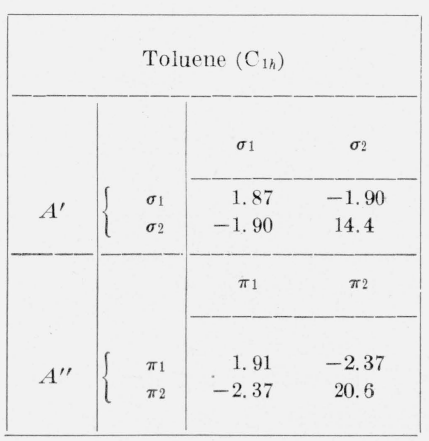

\begin{tabular}{|c|cccc|}
\hline \multicolumn{5}{c|}{ Ethylbenzene $\left(\mathrm{C}_{1 h}\right)$} \\
\hline \\
\hline
\end{tabular}


As stated previously, the intermediate steps in the calculation are dependent upon the choice of $x, y, z$-axes, but the final kinetic-energy matrices (after removal of over-all rotation) are not. The calculations for propane are described in some detail in order to illustrate the method, and the choice of axes for propane will therefore be stated. The $x$ - and $z$-axes were taken in the plane of the $\mathrm{C}-\mathrm{C}-\mathrm{C}$ angle, with the origin at the vertex of the angle, and the $z$-axis bisecting the angle. The $z$-axis therefore remains a twofold axis of symmetry as the $\mathbf{C}-\mathrm{C}-\mathrm{C}$ angle is deformed. The origin is not at the center of mass of the molecule.

The coordinate $\alpha$ in propane is most easily treated as follows. By definition the z-axis bisects the $\mathrm{C}-\mathrm{C}-\mathrm{C}$ angle. Denote the changes in these two half-angles by $\alpha_{1}$ and $\alpha_{2}$. The kinetic-energy matrix is first set up for $\alpha_{1}$ and $\alpha_{2}$, and then the following transformation is carried out:

$$
\left.\begin{array}{l}
\alpha=\left(\alpha_{1}+\alpha_{2}\right) \\
0=\left(\alpha_{1}-\alpha_{2}\right)
\end{array}\right\} .
$$

The second equation is merely the condition that the $z$-axis bisect the $\mathrm{C}-\mathrm{C}-\mathrm{C}$ angle when the latter is deformed. The elements in the kineticenergy matrix for $\left(\alpha_{1}-\alpha_{2}\right)$ are then ignored. The nonorthogonal matrix of this transformation is

$$
\left[\begin{array}{rr}
1 & 1 \\
1 & -1
\end{array}\right] .
$$

Equation 26 leads to the relations

$$
\begin{aligned}
& (\alpha, \alpha)=(1 / 4)\left[\left(\alpha_{1}, \alpha_{1}\right)+2\left(\alpha_{1}, \alpha_{2}\right)+\left(\alpha_{2}, \alpha_{2}\right)\right] \\
& (\alpha, q)=(1 / 2)\left[\left(\alpha_{1}, q\right)+\left(\alpha_{2}, q\right)\right],
\end{aligned}
$$

where $q$ is any coordinate other than $\alpha_{1}, \alpha_{2}$, or $\alpha$.

The following remarks apply to the calculation of the elements of the unreduced kinetic-energy matrix, eq 40, for propane. If the origin of the $\xi, \eta, \zeta$-axes (the point $x_{o r}, y_{o r}, z_{o r}$ ) is located at the carbon atom on the $\xi$-axis (or at any point on the $\xi$-axis for the balanced rotations $\phi_{1}, \phi_{2}$, and $\left.\phi_{3}\right)$, then $I_{\xi \eta}$ and $I_{\zeta \xi}$, vanish in each case, and $H_{x r}^{(1)}$, $H_{y r}^{(1)}$, and $H_{z r}^{(1)}$ may be calculated from eq 61 . $H_{x r}^{(2)}, H_{y r}^{(2)}$, and $H_{z r}^{(2)}$ vanish for the balanced rota- tions $\phi_{1}, \phi_{2}$, and $\phi_{3}$. The diagonal terms $K_{r r}$ are obtained from eq 68. For balanced rotations $K_{r r}=I_{\xi}$. Coordinates with different subscripts (1, 2, or 3) are unconnected, and $K_{r, r^{\prime}}$ is calculated from eq 69. $K_{r, r^{\prime}}$ vanishes if either rotation is balanced ( $\phi$-coordinates), or if the directions of motion of the centers of mass are perpendicular (thus $\pi$-coordinates are perpendicular to $\sigma$ - and $\alpha$-coordinates). Coordinates with the same subscripts are connected, and $K_{r\left(r^{\prime}\right)}$ is obtained from eq 70 (the first term being given by eq 74). From a consideration of the directions of the $\xi$-axes, and the directions of motion of the centers of mass, for each coordinate, it is readily seen that the only terms that do not vanish are $K_{\alpha i(\sigma i)}$ $(i=1,2)$.

The numerical values of the elements of the matrices of eq 40 (one matrix for each representation) are given for propane in table 2 .

The next step is the removal of the over-all rotations by the reduction process of eq 16 . In the subsequent calculations the frequencies of the $\phi_{1}$ and $\phi_{2}$ internal coordinates (methyl rotations in propane and toluene, and methyl and ethyl rotations in ethylbenzene) have been assumed to be zero. This is justifiable because the potential barriers for these rotations ${ }^{10}$ correspond to sufficiently low frequencies for small oscillations from the equilibrium configuration. The coordinates $\phi_{1}$ and $\phi_{2}$ have, therefore, also been removed by the reduction process of eq 16 .

The final reduced kinetic-energy matrices, one for each representation, for each molecule, are shown in table 3.

\section{Calculation of Frequencies}

The force constants are now to be evaluated from the observed frequencies of propane and toluene. These force constants may then be substituted in the secular equation for ethylbenzene to yield calculated frequencies for the latter molecule.

The frequency assignment for propane has been taken from Pitzer [9], and the assignment for toluene from Pitzer and Scott [10], The frequencies $(\nu)$ are summarized in table 4 . The

${ }_{10}$ Propane, reference [9]; toluene and ethylbenzene, reference [11]. 
normal coordinates are mixtures of the coordinates in each representation, and when this mixing is appreciable it is not possible to assign the frequencies in each representation to the coordinates without some ambiguity. However, the pairing indicated is fairly certain. Previous investigators have assigned the two $B_{2}$ frequencies as $\pi^{-}$(748) and $\pi_{3}$ (1179), but the assignment of these frequencies in table 4 is supported by (1) the force constants obtained for $\phi_{3}, \sigma_{3}$, and $\pi_{3}$, which should have approximately the proportions 2,1 , and 1 , respectively (these figures assume that interaction constants are negligible, and that the main constants arise from the constants for the four $C-C-H$ angles), and (2) the approximate equality of the force constants obtained for $\pi^{-}$ and $\sigma^{-}$.

TABLE 4.-Frequencies and calculated force constants for propane and toluene

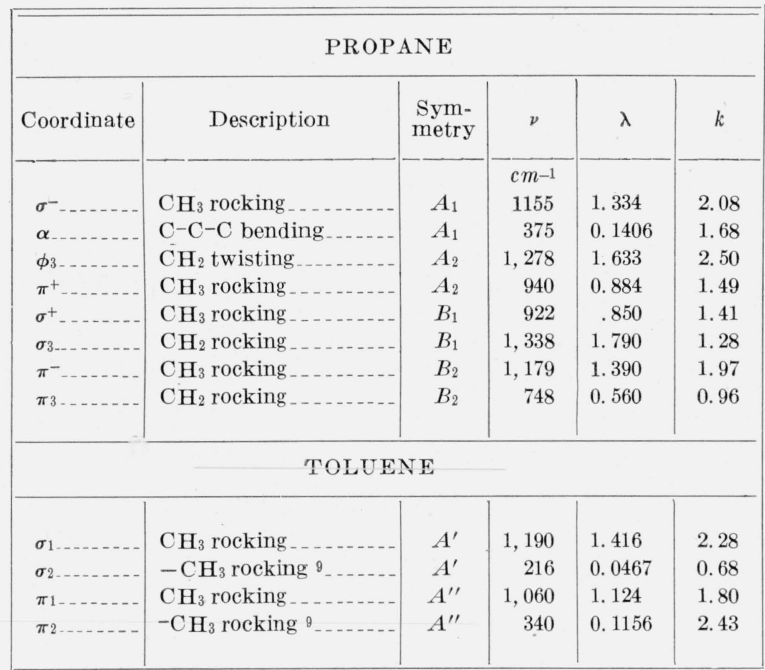

The next column of table 4 gives the value of $\lambda$ (eq 22 to 24 ) in the units

$$
\lambda=\left[\nu\left(\mathrm{cm}^{-1}\right) / 1000\right]^{2} .
$$

The last column shows the calculated values for the force constants, $k$, as calculated from the secular equations. They are the diagonal elements in the potential energy, or $F$, matrix (off-diagonal elements have been set equal to zero as there are not sufficient data to determine them). The units of these force constants corres- pond to the units that have been used for $\lambda$ and for the kinetic-energy matrices in table 3 ; in the reverse calculation for ethylbenzene the frequencies are again obtained in $\mathrm{cm}^{-1}$.

Table 5 shows the force constants selected for the ethylbenzene molecule. Those derived from toluene are $\sigma_{2}$ from $\sigma_{2}$, and $\pi_{2}$ from $\pi_{2}$. Those derived from propane are $\sigma_{1}$ from $\left(\sigma^{+}+\tau^{-}\right) / 2$, $\pi_{1}$ from $\left(\pi^{+}+\pi^{-}\right) / 2$, and $\sigma_{3}, \pi_{3}, \phi_{3}$ and $\alpha$ from $\sigma_{3}$, $\pi_{3}, \phi_{3}$, and $\alpha$, respectively. The averaged force constants, $\left(\sigma^{+}+\sigma^{-}\right) / 2$ and $\left(\pi^{+}+\pi^{-}\right) / 2$, are the main force constants for methyl rocking in propar The observed separation of the frequencies lui $\sigma^{+}, \sigma^{-}$, and for $\pi^{+}, \pi^{-}$, in propane, is due to methylmethyl interaction constants and the twofold symmetry.

TABLE 5.-Force constants and calculated frequencies for ethylbenzene

\begin{tabular}{|c|c|c|c|c|c|}
\hline \multicolumn{6}{|c|}{ ETHYLBENZENE } \\
\hline Coordinate & Description & $\begin{array}{l}\text { Sym- } \\
\text { metry }\end{array}$ & $k$ & $\lambda$ & $\nu$ \\
\hline & $\mathrm{CH}_{3}$ rocking & $A^{\prime}$ & 1.75 & 1.067 & $\begin{array}{l}c m-1 \\
1.035\end{array}$ \\
\hline$\sigma_{2} \ldots$ & $\mathrm{C}_{2} \mathrm{H}_{5}$ rocking $\ldots$ & $A^{\prime}$ & 0.68 & 0.0187 & 137 \\
\hline$\sigma_{3} \ldots$ & $\mathrm{CH}_{2}$ rocking .... & $A^{\prime}$ & 1. 28 & 1. 721 & 1,310 \\
\hline$\alpha \ldots$ & $\mathrm{C}-\mathrm{C}-\mathrm{C}$ bending & $A^{\prime}$ & 1. 68 & 0.1437 & 380 \\
\hline$\phi_{3} \ldots$ & $\mathrm{CH}_{2}$ twisting $\ldots$ & $A^{\prime \prime}$ & 2.50 & 1.615 & 1,270 \\
\hline$\pi_{1} \ldots$ & $\mathrm{CH}_{3}$ rocking $\ldots$ & $A^{\prime \prime}$ & 1. 73 & 1.144 & 1,070 \\
\hline$\pi_{2} \ldots$ & $\mathrm{C}_{2} \mathrm{H}_{5}$ rocking & $A^{\prime \prime}$ & 2.43 & 0.1025 & 320 \\
\hline$\pi_{3} \ldots$ & $\mathrm{CH}_{2}$ rocking $\ldots \ldots$ & $A^{\prime \prime}$ & 0.96 & .604 & 775 \\
\hline
\end{tabular}

Substitution of these force constants in the secular equations for ethylbenzene then yields the values of $\lambda$, and the calculated frequencies, shown in the last two columns of table 5. The observed frequencies for ethylbenzene are discussed in the next section.

\section{Vibrational Frequencies of Ethylbenzene}

The present calculations were undertaken as an aid to the assignment of the vibrational frequencies of ethylbenzene. * These frequencies were required for the calculation of the thermodynamic functions by statistical methods [11]. The frequencies have been assigned on a semiempirical basis which involved a detailed consideration of only the

*F. G. Brickwedde, M. Moskow, and R. B. Scott [24] have also presented an assignment of the vibrational frequencies of ethylbenzene. 
lowest frequencies of vibration of the benzene ring and also of the frequencies associated with the vibration of the ethyl group. The remaining frequencies $\mathrm{w}$ re taken from the assignment for toluene [10]. A complete frequency assignment for ethylbenzene at this time is impracticable.

Several workers have reported Raman spectra $[18,4,15,16,17,18]$ and infrared spectra [19, $20,21,22,23]$ for ethylbenzene. The lowest observed frequencies in the Raman spectrum of ethylbenzene, are, approximately, 154, 305 (weak) 99 (weak), 452, 485, 538 (weak), 553 (weak), and $620 \mathrm{~cm}^{-1}$. The frequencies 480, 567, and $617 \mathrm{~cm}^{-1}$ have been reported in the infrared spectrum in this region. It will be helpful to list the six lowest frequencies of toluene, as assigned by Pitzer and Scott [10]: 11, 216 (R); 18b, 340 (R); 16a, 405 (R) ; 16b, 467 (R); 6a, 521 (R); and $6 \mathrm{~b}, 622$ (R, IR). The first number designates the mode of vibration (see fig. 6, of Pitzer and Scott), the second is the frequency in $\mathrm{cm}^{-1}$, and $\mathrm{R}$ and IR refer to the presence of the line in the Raman or the infrared spectrum, respectively.

The vibrations 11 and $18 \mathrm{~b}$ correspond principally to the rocking of the alkyl group perpendicular to and parallel to the plane of the benzene ring, respectively. The frequencies of these modes in ethylbenzene were calculated as $137 \mathrm{~cm}^{-1}$ for 11 and $320 \mathrm{~cm}^{-1}$ for $18 \mathrm{~b}$ by means of the approximate normal coordinate analysis of section III (these vibrations were denoted by $\sigma_{2}$ and $\pi_{2}$, respectively, in that section). The only possibility for the assignment of the $154 \mathrm{~cm}^{-1}$ frequency is therefore mode 11 . The mode $18 \mathrm{~b}$ was assigned the calculated frequency of $320 \mathrm{~cm}^{-1}$. The frequency observed at $305 \mathrm{~cm}^{-1}$ may easily be the overtone of $154 \mathrm{~cm}^{-1}$.

The mode 16a has a frequency of $400 \mathrm{~cm}^{-1}$ in benzene and $405 \mathrm{~cm}^{-1}$ in toluene. The observed frequency at $399 \mathrm{~cm}^{-1}$ in ethylbenzene may represent $16 \mathrm{a}$, or an overlap of $16 \mathrm{a}$ with the carbon skeletal bending in the ethyl group, which should fall at about $380 \mathrm{~cm}^{-1}$. The toluene value, $405 \mathrm{~cm}^{-1}$, was retained for ethylbenzene. The mode $16 \mathrm{~b}$ has the frequency $467 \mathrm{~cm}^{-1}$ in toluene; the nearest observed frequency in the spectrum of ethylbenzene, $452 \mathrm{~cm}^{-1}$, was assigned to this mode. The mode $6 \mathrm{a}$ in toluene gives rise to a relatively intense line at $521 \mathrm{~cm}^{-1}$. The variation of the frequency of the mode $6 \mathrm{a}$ in other monosubstituted benzenes, as a function of the mass of the attached group [12], indicates that 6 a should be lowered somewhat in ethylbenzene, since the effective mass of the ethyl group in this mode is considerably greater than that of a methyl group. Therefore, the frequency $485 \mathrm{~cm}^{-1}$, which is the strongest observed line in this region, was assigned to $6 \mathrm{a}$. The mode $6 \mathrm{~b}$ has a frequency of $606 \mathrm{~cm}^{-1}$ in benzene, $622 \mathrm{~cm}^{-1}$ in toluene, and remains essentially unchanged in frequency in other monosubstituted benzene [12]. The frequency observed in ethylbenzene at $620 \mathrm{~cm}^{-1}$ was therefore assigned to $6 \mathrm{~b}$.

The mode $7 \mathrm{a}$, which is principally the stretching of the $\mathrm{C}-\mathrm{C}$ bond joining the alkyl group to the ring, has a frequency of $1,210 \mathrm{~cm}^{-1}$ in toluene. As a strong line appears at about $1,200 \mathrm{~cm}^{-1}$ in the ethylbenzene Raman spectrum, the toluene frequency was retained for $7 \mathrm{a}$. The other $\mathrm{C}-\mathrm{C}$ stretching mode in the ethyl group was assigned the frequency of the strong line at $965 \mathrm{~cm}^{-1}$ in the Raman spectrum of ethylbenzene as this is close to the average of the two carbon-carbon stretching frequencies of 868 and $1,053 \mathrm{~cm}^{-1}$ in propane [9]. The symmetry that splits these two components in propane is absent in ethylbenzene.

The symmetrical hydrogen bending in the methyl group was assigned the frequency 1,385 $\mathrm{cm}^{-1}$, and the doubly degenerate unsymmetrical bending the frequency $1,460 \mathrm{~cm}^{-1}$. The symmetrical hydrogen bending in the methylene group was assigned the value $1,460 \mathrm{~cm}^{-1}$. The value $2,960 \mathrm{~cm}^{-1}$ was assigned the $\mathrm{C}-\mathrm{H}$ stretchings in the methyl and methylene groups. These assignments were made on the basis of the frequencies of these modes in propane and toluene, and to some extent on the basis of the spectrum of ethylbenzene.

The remaining frequencies of vibration of the ethyl group were assigned values calculated by means of the approximate normal coordinate analysis of section III (see tables 1 and 5).

The complete frequency assignment for ethylbenzene is shown in table 6 . 
TABLE 6.-Vibration frequencies of the ethylbenzene molecule

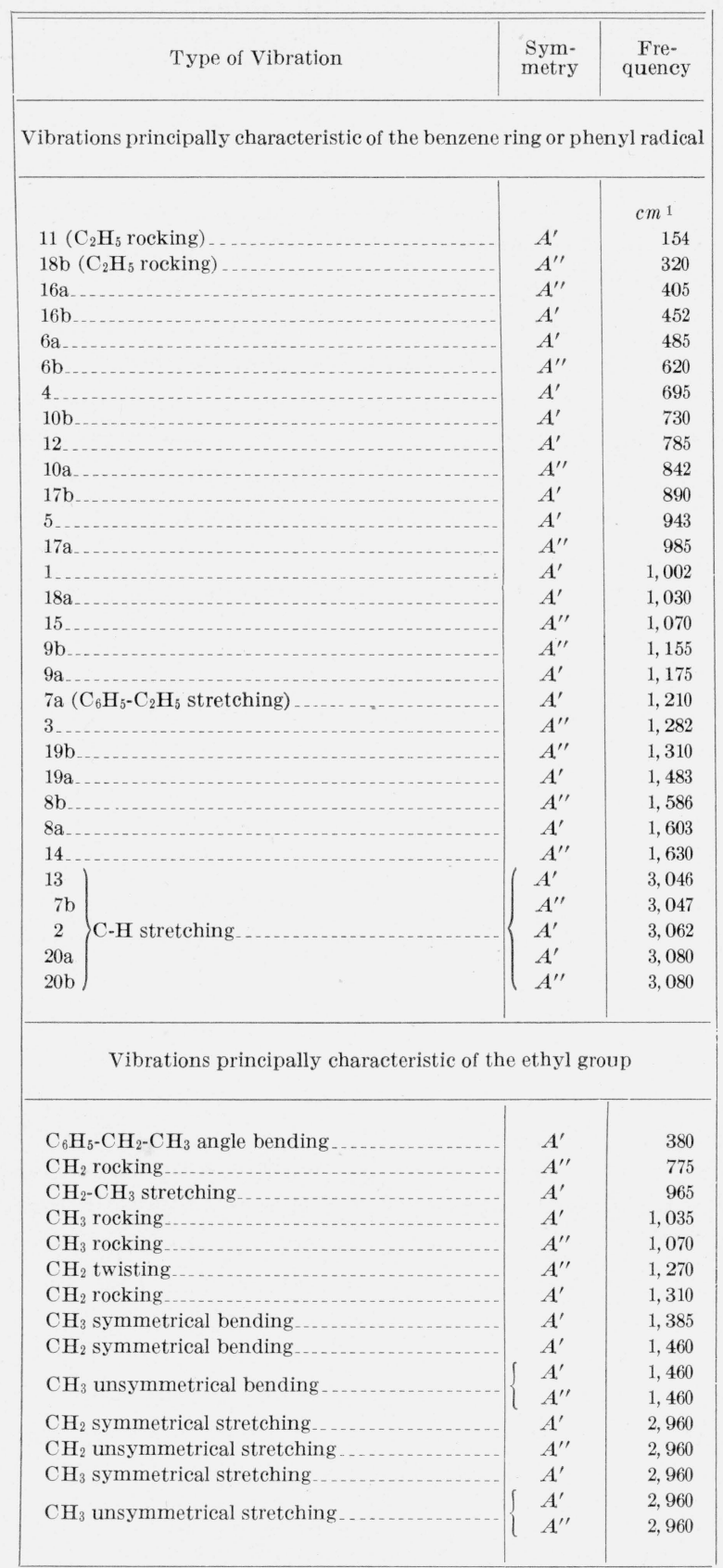

\section{References}

[1] L. S. Majantz, Compt. rend. URSS 32, 119 (1941).

[2] A. Sayvetz, J. Chem. Phys. \%, 383 (1939).

[3] B. L. Crawford, Jr., and E. B. Wilson, Jr., J. Chem. Phys. 9, 323 (1941).

[4] E. B. Wilson, Jr., J. Chem. Phys. y, 1047 (1939); 9, 76 (1941).

[5] A. G. Meister and F. F. Cleveland, Am. J. Phys. 14, 13 (1946).
[6] J. E. Rosenthal and G. M. Murphy, Rev. Modern Phys. 8, 317 (1936).

[7] S. Silver, J. Chem. Phys. 8, 919 (1940).

[8] L. Pauling and L. O. Brockway, J. Am. Chem. Soc. 59, 1223 (1937).

[9] K. S. Pitzer, J. Chem. Phys. 12, 310 (1944).

[10] K. S. Pitzer and D. W. Scott, J. Am. Chem. Soc. 65, 803 (1943).

[11] W. J. Taylor, D. D. Wagman, Mary G. Williams, K. S. Pitzer, and F. D. Rossini, J. Research NBS 37, 95 (1946) RP1732.

[12] K. W. F. Kohlrausch, Der Smekal-Raman-Effekt, p. 163 (J. Springer, Berlin, 1938).

[13] A. Dadieu and K. W. F. Kohlrausch, Monatsh. 53-54, 282 (1929).

[14] J. Soderqvist, Z. Physik 59, 446 (1930).

[15] A. S. Ganesan and S. Venkateswaran, Indian J. Phys. 13, 195 (1929).

[16] R. Signer and J. Weiler, Helv. Chim. Acta. 15, 649 (1932).

[17] A. W. Reitz and W. Stockmair, Monatsh. 67, 92 (1935).

[18] G. Dupont, R. Dulou, and V. Desreux, Bul. soc. chim. 3, 1639 (1936).

[19] P. Lambert and J. Lecomte, Ann. phys. 18, 329 (1932).

[20] R. Stair and W. W. Coblentz, J. Research NBS 15, 295 (1935) RP830.

[21] P. Lambert and J. Lecomte, Ann. Phys. 10, 503 (1938).

[22] American Petroleum Institute Research Project 44 at the National Bureau of Standards. Catalog of infrared absorption spectrograms. Ethylbenzene: Serial No. 53, contributed by the Phillips Petroleum Co. and University of Oklahoma Research Institute; Serial Nos. 123 and 194, contributed by the Texas Co.; Serial No. 135, contributed by the Mellon Institute of Industrial Research and Carnegie Institute of Technology; Serial No. 309, contributed by the U. S. Naval Research Laboratory.

[23] Shell Development Co., Emeryville, Calif., Unpublished data, made available by Otto Beeck.

[24] F. G. Brickwedde, M. Moskow, and R. B. Scott, J. Chem. Phys. 13, 547 (1945).

\section{Appendix $1^{11}$}

Let the coordinates $q_{1}$ be divided into two groups, $q_{a}$ and $q_{b}$, and partition $S_{11}$ into the corresponding submatrices. Then the kinetic energy matrix, eq 4 , becomes

$$
\left[\begin{array}{ccc}
S_{a a} & S_{a b} & S_{a 2} \\
\tilde{S}_{a b} & S_{b b} & S_{b 2} \\
\tilde{S}_{a 2} & \tilde{S}_{b 2} & S_{22}
\end{array}\right] .
$$

The removal of the cross terms in the kinetic energy that contain $\dot{q}_{a}$ leads to the reduced matrix

$$
\left[\begin{array}{ll}
\left(S_{b b}-\tilde{S}_{a b} S_{a a}^{-1} S_{a b}\right) & \left(S_{b 2}-\tilde{S}_{a b} S_{a a}^{-1} S_{a 2}\right) \\
\left(\tilde{S}_{b 2}-\tilde{S}_{a 2} S_{a a}^{-1} S_{a b}\right) & \left(S_{22}-\tilde{S}_{a 2} S_{a a}^{-1} S_{a 2}\right)
\end{array}\right],
$$

and the removal of the remaining cross terms that contain $\dot{q}_{b}$ leads to the final reduced matrix for the coordinates $q_{2}$,

11 See section II, 1 (following eq. 16). 


$$
\begin{aligned}
S_{22}^{\prime}= & \left(S_{22}-\tilde{S_{a 2}} S_{a a}^{-1} S_{a 2}\right)- \\
& \left(\tilde{S_{32}}-\tilde{S_{a 2}} S_{a a}^{-1} S_{a b}\right)\left(S_{b b}-\tilde{S_{a b}} S_{a a}^{-1} S_{a b}\right)^{-1} \\
& \left(S_{b 2}-\tilde{S_{a b}} S_{a a}^{-1} S_{a 2}\right) .
\end{aligned}
$$

Equation 81 may be rewritten in the form

$$
\begin{gathered}
S_{22}^{\prime}=S_{22}-\left[\tilde{S}_{a 2} \tilde{S}_{b 2}\right]\left[\begin{array}{c}
\left(S_{a a}^{-1}+S_{a a}^{-1} S_{a b} G_{b b}^{\prime} \tilde{S}_{a b} S_{a a}^{-1}\right) \\
-G_{b b}^{\prime} \tilde{S}_{a b} S_{a a}^{-1} \\
-S_{a a}^{-1} S_{a b} G_{b b}^{\prime} \\
G_{b b}^{\prime}
\end{array}\right]\left[\begin{array}{c}
S_{a 2} \\
S_{b 2}
\end{array}\right],
\end{gathered}
$$

where

$$
G_{b b}^{\prime}=\left(S_{b b}-\tilde{S}_{a b} S_{a a}^{-1} S_{a b}\right)^{-1}
$$

On the other hand, the simultaneous removal of the cross terms that involve $\dot{q}_{a}$ and $\dot{q}_{b}$ leads to the reduced matrix

$$
S_{22}^{\prime}=S_{22}-\left[\begin{array}{ll}
\tilde{S}_{a 2} & \tilde{S}_{b 2}
\end{array}\right]\left[\begin{array}{ll}
S_{a a} & S_{a b} \\
\tilde{S}_{a b} & S_{b b}
\end{array}\right]^{-1}\left[\begin{array}{l}
S_{a 2} \\
S_{b 2}
\end{array}\right]
$$

The matrices $S_{22}^{\prime}$ calculated from eq 82 and 84 are identical, provided that

$$
\begin{aligned}
& {\left[\begin{array}{cc}
\left(S_{a a}^{-1}+S_{a a}^{-1} S_{a b} G_{b b}^{\prime} \tilde{S}_{a b} S_{a a}^{-1}\right) & -S_{a a}^{-1} S_{a b} C_{b b}^{\prime} \\
-G_{b b}^{\prime} \tilde{S}_{a b} S_{a a}^{-1} & G_{b b}^{\prime}
\end{array}\right]} \\
& {\left[\begin{array}{cc}
S_{a a} & S_{a b} \\
\tilde{S}_{a b} & S_{b \vdash}
\end{array}\right]=\left[\begin{array}{ll}
E_{a a} & 0 \\
0 & E_{b \downarrow}
\end{array}\right]}
\end{aligned}
$$

where $E_{a a}$ and $E_{b b}$ are unit matrices. But the last relation is easily verified by multiplying out the matrices on the left. This establishes the theorem when $q_{1}$ is divided into two groups; the truth of the theorem for any number of groups follows by induction.

\section{Appendix $2{ }^{12}$}

From the relation that $S G=E$, where $E$ is the unit matrix, and eq 3 , there are obtained the relations

$$
\begin{aligned}
& \tilde{S}_{12} G_{12}+S_{22} G_{22}+S_{23} \tilde{G}_{23}=E \\
& S_{11} G_{12}+S_{12} G_{22}+S_{13} \tilde{G}_{23}=0 \\
& S_{12} G_{13}+S_{22} G_{23}+S_{23} G_{33}=0 \\
& S_{11} G_{13}+S_{12} G_{23}+S_{13} G_{33}=0
\end{aligned}
$$

Now multiply eq 89 from the left by $\tilde{S}_{12} S_{11}^{-1}$, and from the right by $G_{33}^{-1} \widetilde{G}_{23}$, and add the resulting equation to eq 86 ; next multiply eq 87 from the left by $\tilde{S}_{12} S_{11}^{-1}$ and eq 88 from the right by $G_{33}{ }^{-1} \tilde{G}_{23}$, and subtract both of the result ing equations from the previous sum. The resulting equation may be factored as follows:

$$
\left(S_{22}-\tilde{S}_{12} S_{11}^{-1} S_{12}\right)\left(G_{22}-G_{23} G_{33}^{-1} \tilde{G}_{23}\right)=E_{22}
$$

Equation 21 follows immediately from eq 16, 20, and 90 .

Washington, September 24, 1946.

12 See section II, 1 (eq 20 and 21 ). 